Supporting Informations

\title{
A Simple One-Flask Method for the Preparation of Hydroxamic Acids.
}

Giampaolo Giacomelli,* Andrea Porcheddu and Margherita Salaris

\section{Experimental Section}

The $N$-protected amino acids were prepared according standard methods and their purities were established before utilization by melting point and optical rotation. ${ }^{1}$ The $N$-protected dipeptide, $N$-BocLeu-Gly-OH and $\mathrm{N}$-Cbz-Pro-Gly-OH were prepared on solid phase. ${ }^{2}$ Cyanuric chloride was purchased from Aldrich while the $N$-protected amino acids were purchase from Fluka. Elemental analyses were performed on a Perkin-Elmer 420 B analyser, optical rotations were measured with a Perkin-Elmer 241 automatic polarimeter in a $1 \mathrm{dm}$ tube. The ${ }^{1} \mathrm{H}$ NMR (300 MHz), ${ }^{13} \mathrm{C}$ NMR $(75.4 \mathrm{MHz})$ and ${ }^{19} \mathrm{~F}$ NMR $(282.3 \mathrm{MHz})$ were obtained with a Varian VXR-300 spectrometer from $\mathrm{CDCl}_{3}$ solutions.

The procedure for the preparation of $(S)$-( $N$-benzyloxycarbonyl)proline hydroxamate, 3 , is representative for all cases. Cyanuric chloride $(0.5 \mathrm{~g}, 3.0 \mathrm{mmol})$ was added to a solution of $(S)-(N-$ benzyloxycarbonyl)proline (2.24 g, $9.0 \mathrm{mmol}), \operatorname{NMM}(1.0 \mathrm{~g}, 1.1 \mathrm{~mL}, 9.9 \mathrm{mmol})$, DMAP $(0.01 \mathrm{~g}, 0.1$ mmol) in $\mathrm{CH}_{2} \mathrm{Cl}_{2}(20 \mathrm{~mL})$, containing hydroxylamine hydrochloride $(0.68 \mathrm{~g}, 9.8 \mathrm{mmol})$, stirred and maintained at $0^{\circ} \mathrm{C}$. After the addition, the mixture was warmed to room temperature, stirred for $12 \mathrm{~h}$, then filtered on celite, and the organic phase washed threefold with $15 \mathrm{~mL}$ of $1 \mathrm{~N} \mathrm{HCl}$ and then brine. The organic layer were dried ( $\left.\mathrm{Na}_{2} \mathrm{SO}_{4}\right)$ and the solvent evaporated to yield compound $\mathbf{3}$ that was isolated without other purifications $(2.3 \mathrm{~g}, 96 \%),[\alpha]^{25} \mathrm{D}-46.1$ ( $c$ 0.5, methanol). ${ }^{1} \mathrm{H}$ NMR $\delta, 8.06$ (s, 1H), 7.26-7.36 (m, 5H), $5.34(\mathrm{~s}, 2 \mathrm{H}), 4.29(\mathrm{t}, 1 \mathrm{H}), 3.35(\mathrm{t}, 2 \mathrm{H}), 2.17(\mathrm{~s}, 1 \mathrm{H}), 1.60-1.83(\mathrm{~m}, 4 \mathrm{H}) ;{ }^{13} \mathrm{C}$ NMR $\delta, 170.0,155.8,137.0,128.4,127.8,127.5,67.0,60.2,40.9,23.7,20.1$.

Benzoic hydroxamate, 1: (1.17 g, 95\%), m. p. $123-124^{\circ} \mathrm{C} .{ }^{3}$

Phenylacetic hydroxamate, 2: (1.22 g, 90\%), oil; ${ }^{1} \mathrm{H}$ NMR $\delta, 8.09$ ( s, 1H), 7.26-7.33 (m, 5H), 3.65 (s, 2H), 2.17 (s, 1H); ${ }^{13} \mathrm{C}$ NMR $\delta, 165.8,129.7,128.8,127.5,35.7$.

\footnotetext{
${ }^{1}$ Falorni, M.; Porcheddu, A.; Taddei, M. Tetrahedron Lett. 1999, 40, 4395.

${ }^{2}$ Falchi, A.; Giacomelli, G.; Porcheddu, A.; Taddei, M. Synlett 2000, 275.

${ }^{3}$ Reddy, A. S.; Kumar, M. S.; Reddy, G. R. Tetrahedron Lett. 2000, 41, 6285.
} 
$(S)-N$-(Benzyloxycarbonyl)alanine hydroxamate, 4: [da $(S)-N$-(benzyloxycarbonyl)alanine, $[\alpha]^{20}{ }_{\mathrm{D}}$ -14.5 (c 1, AcOH): $1.86 \mathrm{~g}, 87 \%$ ], oil, $[\alpha]^{25}{ }_{\mathrm{D}}+10.4$ (c 1, methanol). ${ }^{1} \mathrm{H}$ NMR $\delta, 8.06$ (s, 1H), 7.26-7.33 (m, 5H), 5.34 (s, 2H), 4.71 (q, 1H), $2.17(\mathrm{~s}, 1 \mathrm{H}), 1.48(\mathrm{~d}, 3 \mathrm{H}) ;{ }^{13} \mathrm{C}$ NMR $\delta, 170.0,155.8,137.0,128.4$, 127.8, 127.5, 69.1, 50.8, 17.5. Anal. Calcd. for $\mathrm{C}_{11} \mathrm{H}_{14} \mathrm{~N}_{2} \mathrm{O}_{4}$ (238.24): C, 55.46; H, 5.92; N, 11.76. Found C, 55.49, H, 5.91, N, 11.76.

(S)-N-(Benzyloxycarbonyl)valine hydroxamate, 5: [da $(S)-N$-(benzyloxycarbonyl)valine, $[\alpha]^{20}{ }_{\mathrm{D}}-3.8$ (c 2, AcOH): $2.25 \mathrm{~g}, 94 \%$ ), oil, $[\alpha]^{25} \mathrm{D}-30.5$ (c 1, methanol). ${ }^{1} \mathrm{H}$ NMR $\delta, 8.07$ (bs, 1H), 7.31-7.36 (m, 5H), $5.42(\mathrm{~d}, 1 \mathrm{H}), 5.11(\mathrm{~s}, 2 \mathrm{H}), 4.34(\mathrm{dd}, 1 \mathrm{H}), 2.24(\mathrm{~m}, 1 \mathrm{H}), 2 . .07(\mathrm{~s}, 1 \mathrm{H}), 0.98(\mathrm{~d}, 6 \mathrm{H}) ;{ }^{13} \mathrm{C} \mathrm{NMR} \delta$, 170.0, 156.9, 140.0, 128.6, 127.5, 127.1, 70.0, 60.2, 28.0, 16.2. Anal. Calcd. for $\mathrm{C}_{13} \mathrm{H}_{18} \mathrm{~N}_{2} \mathrm{O}_{4}$ (266.29): C, 58.63; H, 6.81; N, 10.52. Found C, 58.69, H, 6.78, N, 10.52.

$(S)-N$-(Benzyloxycarbonyl)serine hydroxamate, 6: [da $(S)-N$-(benzyloxycarbonyl)serine, $[\alpha]^{20}{ }_{\mathrm{D}}+6.3$ $(c 7, \mathrm{AcOH}): 2.06 \mathrm{~g}, 90 \%]$, oil, $[\alpha]^{25} \mathrm{D}+15.87$ (c 1, methanol). ${ }^{1} \mathrm{H}$ NMR $\delta, 8.06(\mathrm{~s}, 1 \mathrm{H}), 7.24-7.33(\mathrm{~m}$, $5 \mathrm{H}), 5.34(\mathrm{~s}, 2 \mathrm{H}), 4.31(\mathrm{t}, 1 \mathrm{H}), 3.99(\mathrm{~m}, 2 \mathrm{H}), 2.17(\mathrm{~s}, 1 \mathrm{H}) ;{ }^{13} \mathrm{C}$ NMR $\delta, 170.0,155.8,137.0,128.4$, 127.8, 127.5, 69.1, 50.8, 17.5. Anal. Calcd. for $\mathrm{C}_{11} \mathrm{H}_{14} \mathrm{~N}_{2} \mathrm{O}_{5}$ (254.24): C, 51.97; H, 5.55; N, 11.02. Found C, 51.99, H, 5.51, N, 11.04.

$(S)-N$-(Benzyloxycarbonyl)phenylalanine hydroxamate, 7: [da $(S)-N$-(benzyloxycarbonyl)phenylalanine, $\left.[\alpha]^{20}{ }_{\mathrm{D}}+5.0(c 5, \mathrm{AcOH}): 2.60 \mathrm{~g}, 92 \%\right)$, oil, $[\alpha]{ }^{25} \mathrm{D} \cdot-38.8\left(c\right.$ 0.5, methanol). ${ }^{1} \mathrm{H}$ NMR $\delta, 8.09(\mathrm{~s}, 1 \mathrm{H}), 7.26-7.33(\mathrm{~m}, 10 \mathrm{H}), 5.34(\mathrm{~s}, 2 \mathrm{H}), 4.71(\mathrm{q}, 1 \mathrm{H}), 3.84(\mathrm{~m}, 1 \mathrm{H}), 3.56(\mathrm{~m}, 1 \mathrm{H}), 2.07(\mathrm{~s}$, 1H); ${ }^{13} \mathrm{C}$ NMR $\delta, 171.9,155.8,139.8,137.0,129.0,128.4,128.3,127.5,127.3,125.5,69.8,56.7$, 39.0 .4

$(S)-N$-Methyl- $N$-(benzyloxycarbonyl)valine hydroxamate, 8: $(2.42 \mathrm{~g}, 96 \%)$, oil, $[\alpha]{ }_{\mathrm{D}}^{25}+10.0(c)$, methanol). ${ }^{1} \mathrm{H}$ NMR $\delta, 8.07$ (bs, 1H), 7.33-7.36 (m, 5H), 5.28 (s, 2H), 4.35 (dd, 1H), 2.96 (s, 3H), 2.24 $(\mathrm{m}, 1 \mathrm{H}), 2 . .06(\mathrm{~s}, 1 \mathrm{H}), 1.06(\mathrm{~d}, 6 \mathrm{H}) ;{ }^{13} \mathrm{C}$ NMR $\delta, 170.4,157.2,140.0,128.6,127.5,127.1,70.0,59.8$, 33.5, 28.0, 16.5. Anal. Calcd. for $\mathrm{C}_{14} \mathrm{H}_{20} \mathrm{~N}_{2} \mathrm{O}_{4}$ (280.32): C, 59.99; H, 7.19; N, 9.99. Found C, 59.99, H, 7.15, N, 10.00 .

$(S)-N$-(tert-Butoxycarbonyl)proline hydroxamate, 9: [da $(S)-N$-(tert-butoxycarbonyl)proline, $[\alpha]^{20} \mathrm{D}^{-}$ 61.1 (c 1, AcOH): $1.76 \mathrm{~g}, 85 \%]$, oil, $[\alpha]^{25} \mathrm{D}-57.1$ (c 1, methanol). ${ }^{4}{ }^{1} \mathrm{H}$ NMR $\delta, 8.06(\mathrm{~s}, 1 \mathrm{H}), 4.29(\mathrm{t}$, $1 \mathrm{H}), 3.35(\mathrm{t}, 2 \mathrm{H}), 2.17(\mathrm{~s}, 1 \mathrm{H}), 1.60-1.83(\mathrm{~m}, 4 \mathrm{H}), 1,45(\mathrm{~s}, 9 \mathrm{H})$.

\footnotetext{
${ }^{4}$ Thouin, E.; Lubell, W. D.. Tetrahedron Lett. 2000, 41, 457.
} 
(S)- $N$-(tert-Butoxycarbonyl)alanine hydroxamate, 10: [da $(S)$ - $N$-(tert-butoxycarbonyl)alanine, $[\alpha]^{20}{ }_{\mathrm{D}}-25.6$ (c 1, AcOH): $\left.1.65 \mathrm{~g}, 90 \%\right]$, oil, $[\alpha]^{25} \mathrm{D}-36.0$ (c 1, methanol). ${ }^{4}{ }^{1} \mathrm{H}$ NMR $\delta, 8.09(\mathrm{~s}, 1 \mathrm{H}), 4.71(\mathrm{q}, 1 \mathrm{H}), 2.17(\mathrm{~s}, 1 \mathrm{H}), 1.48(\mathrm{~s}, 9 \mathrm{H}), 1.39(\mathrm{~d}, 3 \mathrm{H})$.

(S)- $N$-(tert-Butoxycarbonyl)- $\boldsymbol{O}$-benzyl glutamic acid hydroxamate, 11: (2.74 g, 90\%), m. p. 82$83^{\circ} \mathrm{C},[\alpha]^{25}{ }_{\mathrm{D}}+27.7$ (c 1, methanol). ${ }^{1} \mathrm{H}$ NMR $\delta, 8.06(\mathrm{~s}, 1 \mathrm{H}), 7.26-7.35(\mathrm{~m}, 5 \mathrm{H}), 5.34(\mathrm{~s}, 2 \mathrm{H}), 5.15(\mathrm{t}$, 1H), 2.92 (s, 2H), 2.09 (s, 1H), 1.45 (s, 9H); ${ }^{3} \mathrm{C}$ NMR $\delta, 173.1,170.0,156.44,139.0,128.8,127.9$, 127.2, 68.1, 49.8, 37.8, 28.3. Anal. Calcd. for $\mathrm{C}_{16} \mathrm{H}_{22} \mathrm{~N}_{2} \mathrm{O}_{6}$ (338.36): C, 56.80; H, 6.55; N, 8.28. Found C, 56.85, H, 6.55, N, 8.32.

$(S)-N$-(tert-Butoxycarbonyl)leucine hydroxamate, 12: [da $(S)-N$-(tert-butoxycarbonyl)leucine, $[\alpha]^{20}{ }_{\mathrm{D}}$ -24.9 (c 1, AcOH): $2.15 \mathrm{~g}, 97 \%], \%)$, oil, $[\alpha]^{25}{ }_{\mathrm{D}}-8.6$ (c 1, methanol). ${ }^{1} \mathrm{H}$ NMR $\delta, 8.05$ (s, 1H), $4.43(\mathrm{t}$, 1H), $2.20(\mathrm{~s}, 1 \mathrm{H}), 1.85(\mathrm{~m}, 1 \mathrm{H}), 1.76(\mathrm{dd}, 2 \mathrm{H}), 1.45(\mathrm{~s}, 9 \mathrm{H}), 0.96(\mathrm{~d}, 6 \mathrm{H}) ;{ }^{13} \mathrm{C} \mathrm{NMR} \delta, 170.1,157.9$, 71.0, 51.1, 41.6, 28.5, 23.1, 22.0. Anal. Calcd. for $\mathrm{C}_{11} \mathrm{H}_{22} \mathrm{~N}_{2} \mathrm{O}_{4}$ (246.30): C, 53.64; H, 9.00; N, 11.37. Found C, 53.69, H, 9.05, N, 11.40.

$(S)-N$-(tert-Butoxycarbonyl)phenylalanine hydroxamate, 13: [da $(S)-N$-(tert-butoxycarbonyl)phenylalanine, $[\alpha]^{20}{ }_{\mathrm{D}}+25.2$ (c 1, EtOH): $\left.2.41 \mathrm{~g}, 95 \%\right]$, oil. ${ }^{1} \mathrm{H}$ NMR $\delta, 8.09$ (s, 1H), 7.26$7.33(\mathrm{~m}, 5 \mathrm{H}), 4.13(\mathrm{t}, 1 \mathrm{H}), 3.84(\mathrm{~m}, 1 \mathrm{H}), 3.56(\mathrm{~m}, 1 \mathrm{H}), 2.04(\mathrm{~s}, 1 \mathrm{H}), 1.45(\mathrm{~s}, 9 \mathrm{H}){ }^{4}$

(9-Fluorenylmethoxycarbonyl)glycine, 14: $\left(2.72\right.$ g, 97\%), m. p. $116-118^{\circ} \mathrm{C} .{ }^{1} \mathrm{H}$ NMR $\delta, 8.05(\mathrm{~s}$, 1H), 7.31-7.75 (m, 8H), $5.28(\mathrm{t}, 2 \mathrm{H}), 4.43(\mathrm{~d}, 1 \mathrm{H}), 3.80(\mathrm{~s}, 2 \mathrm{H}), 2.04(\mathrm{~s}, 1 \mathrm{H}) ;{ }^{13} \mathrm{C} \mathrm{NMR} \delta, 166.1,157.7$, 141,8, 136.3, 128.2, 127.6, 126.3, 44.6, 38.0. Anal. Calcd. for $\mathrm{C}_{17} \mathrm{H}_{16} \mathrm{~N}_{2} \mathrm{O}_{4}$ (312.32): C, 65.38; $\mathrm{H}$, 5.16; N, 8.97. Found C, 65.38, H, 5.20, N, 8.98.

$(S)$ - $N$-(9-Fluorenylmethoxycarbonyl)leucine hydroxamate, $15: \quad[$ da $\quad(S)-N-(9-$ fluorenylmethoxycarbonyl)leucine, $[\alpha]^{20}{ }_{\mathrm{D}}-25.8$ (c 1, DMF): $\left.3.25 \mathrm{~g}, 98 \%\right]$, m. p. $114-116^{\circ} \mathrm{C},[\alpha]^{25} \mathrm{D}$ -13.6 (c 1, methanol). ${ }^{1} \mathrm{H}$ NMR $\delta, 8.05(\mathrm{~s}, 1 \mathrm{H}), 7.31-7.75(\mathrm{~m}, 8 \mathrm{H}), 5.28(\mathrm{t} .2 \mathrm{H}), 4.43(\mathrm{~d}, 1 \mathrm{H}), 4.32(\mathrm{t}$. 1H), $2.20(\mathrm{~s}, 1 \mathrm{H}), 1.85(\mathrm{~m}, 1 \mathrm{H}), 1.76(\mathrm{~d}, 3 \mathrm{H}), 0.96(\mathrm{~d}, 6 \mathrm{H}) ;{ }^{13} \mathrm{C} \mathrm{NMR} \delta, 169.7,156.4,143.8,141.1$, 128.2, 127.6, 126.5, 67.9, 46.8, 37.9, 35.5, 23.1, 22.0. Anal. Calcd. for $\mathrm{C}_{21} \mathrm{H}_{24} \mathrm{~N}_{2} \mathrm{O}_{4}(368.43)$ : C, 68.46; H, 6.57; N, 7.60. Found C, 68.50, H, 6.56, N, 7.54.

(S)-N-(9-Fluorenylmethoxycarbonyl)phenylalanine hydroxamate, 16: [da $(S)-N-(9-$ fluorenylmethoxycarbonyl)phenylalanine, $[\alpha]^{20}{ }_{\mathrm{D}}-39.8$ (c 1, DMF): $\left.2.90 \mathrm{~g}, 80 \%\right]$, m. p. $150-152^{\circ} \mathrm{C}$, $[\alpha]^{25} \mathrm{D}-68.9$ (c 1, methanol). ${ }^{1} \mathrm{H}$ NMR $\delta, 8.09(\mathrm{~s}, 1 \mathrm{H}), 7.26-7.76(\mathrm{~m}, 8 \mathrm{H}), 5.28(\mathrm{~d} .2 \mathrm{H}), 4.17(\mathrm{t}, 1 \mathrm{H})$, 
4.13 (d. 1H), 3.70 (d, 2H), 2.04 (s, 1H); ${ }^{13} \mathrm{C}$ NMR $\delta, 170.2,157.2,140.8,137.1,129.2,128.2,127.6$, 127.1, 125.9, 125.3, 73.0, 55.1, 36.9. Anal. Calcd. for $\mathrm{C}_{24} \mathrm{H}_{22} \mathrm{~N}_{2} \mathrm{O}_{4}$ (402.44): C, 71.63; H, 5.51; N, 6.96. Found C, 71.68, H, 5.56, N, 6.94.

$(S)-N$-(tert-Butoxycarbonyl)phenylglycine hydroxamate, 17: $5.0 \mathrm{~g}(19.9 \mathrm{mmol})$ of $(S)-N$-(tertbutoxycarbonyl)phenylglycine, $[\alpha]^{25} \mathrm{D}+145$ ( c 1, ethanol) gave $4.77 \mathrm{~g}, 90 \%$ of 17, m. p. $28-30^{\circ} \mathrm{C}$, $[\alpha]^{25}{ }_{\mathrm{D}}+46.1$ (c 0.2, methanol). ${ }^{1} \mathrm{H}$ NMR $\delta, 7.92(\mathrm{~s}, 1 \mathrm{H}), 7.32(\mathrm{~m}, 5 \mathrm{H}), 5.21(\mathrm{~m}, 1 \mathrm{H}), 1.48(\mathrm{~s}, 9 \mathrm{H}) ;{ }^{13} \mathrm{C}$ NMR $\delta, 167.8,60.8,137.1,129.2,128.2,127.6,79.0,28.2$. Anal. Calcd. for $\mathrm{C}_{13} \mathrm{H}_{18} \mathrm{~N}_{2} \mathrm{O}_{4}$ (266.29): C, 58.63; H, 6.81; N, 10.52. Found C, 58.60, H, 6.85, N, 10.54.

A sample of $17(0.70 \mathrm{~g}, 0.26 \mathrm{mmol})$ and distilled (+)MPTACl $(0.79 \mathrm{~g}, 0.31 \mathrm{mmol})$ were mixed in $\mathrm{CCl}_{4}(1.0 \mathrm{~mL})$ with dry pyridine $(1.0 \mathrm{~mL})$ and allowed to stand for $12 \mathrm{~h}$. Water was then added, and the reaction mixture extracted with ether $(15 \mathrm{~mL})$, washed successively with diluted $\mathrm{HCl}$, saturated $\mathrm{Na}_{2} \mathrm{CO}_{3}$, water and dried $\left(\mathrm{Na}_{2} \mathrm{SO}_{4}\right)$. TLC analysis showed the presence of only a compound. After removal of the solvent under vacuum, the residue was dissolved in $\mathrm{CDCl}_{3}$ for ${ }^{19} \mathrm{~F} \mathrm{NMR}$ analysis $[\delta,-72.1(\mathrm{~s}, \sim 0.01),-72.5(\mathrm{~s}, \sim 0.99)]$.

The same procedure was repeated on a sample of racemic $N-(\mathrm{tert}-$ butoxycarbonyl)phenylglycine hydroxamate $[\delta,-72.1(\mathrm{~s}, 0.5),-72.5(\mathrm{~s}, 0.5)]$.

(S)-N-Boc-Leu-Gly-NHOH, 18; $\left(1.99\right.$ g, 73\%), m. p. $120-122^{\circ} \mathrm{C},[\alpha]^{25} \mathrm{D}-10.7$ (c 1 , methanol). ${ }^{5}{ }^{1} \mathrm{H}$ NMR $\delta, 8.09(\mathrm{bs}, 1 \mathrm{H}), 4.64(\mathrm{t}, 1 \mathrm{H}), 3.95(\mathrm{~s}, 2 \mathrm{H}), 1.85(\mathrm{~m}, 1 \mathrm{H}), 1.68(\mathrm{~m}, 2 \mathrm{H}), 1.36(\mathrm{~s}, 9 \mathrm{H}), 0.86(\mathrm{~d}$, $6 \mathrm{H}) ;{ }^{13} \mathrm{C}$ NMR $\delta, 173.9,167.0,156.1,73.9,53.4,41.4,41.2,28.3,22.8,21.7$.

(S)-N-Cbz-Pro-Gly-NHOH, 19; $\left(2.37\right.$ g, 82\%), viscous oil, $[\alpha]{ }^{25}{ }_{\mathrm{D}}-80.3$ (c 1, methanol). ${ }^{1} \mathrm{H}$ NMR $\delta$, 8.06 (bs, 1H), 7.22-7.26 (m, 5H), 5.07 (s. 2H), 4.31 (t, 1H), 4.05 (s. 2H), 3.45 (t, 2H), 1.96 (m, 2H), 1.75 (m, 2H); ${ }^{13} \mathrm{C}$ NMR $\delta, 174.0,170.0,155.6,136.1,128.4,127.8,67.1,60.2,43.8,41.1,23.3,20.0$. Anal. Calcd. for $\mathrm{C}_{15} \mathrm{H}_{19} \mathrm{~N}_{3} \mathrm{O}_{5}$ (321.33): C, 56.07; H, 5.96; N, 13.08. Found C, 56.11, H, 5.96, N, 13.04.

All compounds 1-19 gave a positive test for hydroxamic acids on treatment with an iron(III) chloride solution. ${ }^{6}$

\footnotetext{
5 Odake, S.; Nakahashi, K., Morikawa, T., Takebe, S.; Kobashi, K. Chem. Pharm. Bull. 1992, 40, 2764.

6 Vogel's Textbook of Practical Organic Chemistry Longman Ed. 5th ed, 1989, p. 1222.
} 